Management international

International Management

Gestiòn Internacional

\title{
L'agriculture durable. Comment réconcilier le public et le privé pour la gestion d'un bien commun
}

\section{Sustainable Agriculture. How to Conciliate Public and Private for a Common Good Management Agricultura sostenible. Como conciliar público y privado para la gestión de un bien común}

\section{Christophe Assens et François Coléno}

Volume 21, numéro 4, été 2017

URI : https://id.erudit.org/iderudit/1053583ar

DOI : https://doi.org/10.7202/1053583ar

Aller au sommaire du numéro

Éditeur(s)

HEC Montréal

Université Paris Dauphine

ISSN

1206-1697 (imprimé)

1918-9222 (numérique)

Découvrir la revue

Citer cet article

Assens, C. \& Coléno, F. (2017). L’agriculture durable. Comment réconcilier le public et le privé pour la gestion d'un bien commun. Management international International Management / Gestiòn Internacional, 21(4), 136-145.

https://doi.org/10.7202/1053583ar

\section{Résumé de l'article}

Face au réchauffement climatique, à l'augmentation de la population mondiale et aux demandes sociétales il est nécessaire de repenser la diffusion des innovations agricoles en impliquant tout les maillons de la chaîne de valeur agro-alimentaire et en leur fournissant un bénéfice équitable. Dans cet article nous présentons la gestion collective d'une filière du fait d'une gestion en bien commun d'une innovation variétale (la pomme Ariane ${ }^{\circledR}$ ). Cette modalité de gestion est permise par un partenariat public-privé original entre un institut de recherche public ('INRA) et les acteurs de la chaîne de valeur.
Tous droits réservés @ Management international / International Management / Gestión Internacional, 2017
Ce document est protégé par la loi sur le droit d'auteur. L'utilisation des services d’Érudit (y compris la reproduction) est assujettie à sa politique d'utilisation que vous pouvez consulter en ligne. 


\section{L'agriculture durable. Comment réconcilier le public et le privé pour la gestion d'un bien commun.}

\section{Sustainable Agriculture. How to Conciliate Public ; and Private for a Common Good Management.}

\section{Agricultura sostenible. Como conciliar público y privado para la gestión de un bien común}

\author{
CHRISTOPHE ASSENS \\ LAREQUOI, ISM, Université de Versailles St Quentin
}

\author{
FRANÇOIS COLÉNO \\ INRA UMR 1048 SADAPT
}

\section{RÉSUMÉ}

Face au réchauffement climatique, à l'augmentation de la population mondiale et aux demandes sociétales il est nécessaire de repenser la diffusion des innovations agricoles en impliquant tout les maillons de la chaîne de valeur agro-alimentaire et en leur fournissant un bénéfice équitable. Dans cet article nous présentons la gestion collective d'une filière du fait d'une gestion en bien commun d'une innovation variétale (la pomme Ariane ${ }^{\circledast}$ ). Cette modalité de gestion est permise par un partenariat public-privé original entre un institut de recherche public (l'INRA) et les acteurs de la chaîne de valeur.

Mots clés : bien commun, agriculture durable, réseau public-privé, innovation, pomme.

\section{ABSTRACT}

In front of global warming of world demography increase, and of social demand it is important to redesign agricultural innovation process involving the stakeholders on the food-processing value chain providing them a fair profit. In this article we analyze the collective management of an agricultural supply chain allowed by the management of an cultivar innovation (the Ariane ${ }^{\star}$ apple) as a common good. Such a management is due to an original public private partnership between a public research institute (INRA) and the member of the value chain.

Keywords: common good, sustainable agriculture, public-private network, innovation, apple.

\section{RESUMEN}

Ante el calentamiento global el aumento de la demografía mundialy de la demanda social, es importante rediseñar el proceso de innovación agrícola involucrando a las partes interesadas en la cadena de valor del procesamiento de alimentos, proporcionándoles un beneficio justo. En este artículo analizamos la gestión colectiva de una cadena de suministro agrícola permitida por la gestión de una innovación de cultivar (la manzana Ariane ${ }^{\varpi}$ ) como bien común. Esta gestión se debe a una original asociación público-privada entre un instituto público de investigación (INRA) y el miembro de la cadena de valor.

Palabras clave: bien común, agricultura sostenible, red pública y privada, innovación, manzana.
T es pertes de production agricoles liées aux bio-agresseurs Lreprésentent près de $40 \%$ de la production mondiale (Savary et al., 2012). La lutte contre ces bio-agresseurs est donc un enjeu primordial au niveau mondial. Cette lutte passe principalement à l'heure actuelle par un recours à des solutions chimiques. Néanmoins, outre l'impact de celles-ci sur l'environnement ces solutions perdent leur efficacité de plus en plus rapidement (McDonald et Linde, 2002). Le recours à des solutions alternatives, plus respectueuses de l'environnement et contribuant à des systèmes de production agricoles permettant d' "assurer une production pérenne de nourriture, de bois et de fibres en respectant les limites écologiques, économiques et sociales qui assurent la maintenance dans le temps de cette production" (United Nations Organization, 1992) est une priorité. Dans ce contexte, l'innovation dans la génétique des semences devient un axe de réflexion majeur pour les entreprises du secteur de l'agroalimentaire, afin de déterminer des variétés de semences qui résistent naturellement aux agressions (insectes, champignons, bactéries...), pour assurer des rendements importants, tout en limitant l'utilisation de pesticides qui sont contre productifs sur le long terme. On parle de gènes de résistance. Lorsque les filières sont de faible taille les acteurs privés seuls ne peuvent se lancer dans des programmes d'amélioration génétique longs et couteux. Dans ces cas, les innovations en génétique des plantes sont liées à des collaborations entre entreprises privées et laboratoires de recherches publics, dispositif qui favorise au mieux le transfert de connaissances entre la recherche fondamentale et la mise en application à des fins économiques (Link et Rees, 1990).

Toutefois, les innovations variétales posent un problème majeur de durabilité, en particulier lorsque les mêmes variétés (ou gènes de résistance) sont utilisées sur une large portion du territoire du fait d'une adaptation rapide des pathogènes et de leur dispersion spatiale. L'efficacité des résistances reste alors trop transitoire (McDonald et Linde, 2002). Une modalité de gestion permettant d'assurer la durabilité des résistances variétales consisterait à mettre en place une diversité génétique des résistances à l'échelle du territoire évitant ainsi durablement l'utilisation de pesticides (Zhu et al., 2000). Une telle modalité de gestion repose sur l'émergence de stratégies collectives visant à assurer la durabilité de la ressource génétique sur le long terme évitant ainsi une tragédie des communs (Hardin, 1968). La prise en compte de la ressource génétique comme un bien commun, et du coups l'émergence de stratégies collectives pour gérer cette ressource ne va pas de soit et rencontre de nombreux échecs. 
Nous présentons dans cet article, l'histoire du regroupement de plusieurs PME sous la forme d'un réseau (Assens, 2013), pour créer, labéliser puis gérer comme un bien commun une nouvelle variété de fruits adaptée au développement durable : la pomme Ariane ${ }^{\circledast}$. L'initiative de cette alliance stratégique revient à un laboratoire public au sein de l'INRA, en position de tiers de confiance, pour favoriser le rapprochement des entreprises privées.

Dans une première partie nous préciserons la notion de bien commun et les formes de gestion de ce type de bien, ni totalement public, ni totalement privé. Dans une seconde partie, le cadre méthodologique présente la manière dont la gestion du bien commun est étudié à partir d'une étude de cas sur l'innovation publique privée dans la filière de la pomme. Dans les parties suivantes, nous présentons les modalités de régulation du bien commun dans l'étude de cas, soit de manière séquentielle par des modalités de régulation publique ou privée, soit de manière simultanée. En conclusion, nous insistons sur cette nécessité de réguler des biens communs, comme l'innovation technologique dans l'agriculture durable, en combinant le rôle du marché et de la puissance publique dans des réseaux hybrides, seuls capables de concilier les objectifs contradictoires de la protection de l'environnement et de l'amélioration des rendements économiques.

\section{Le cadre théorique : le management d'un bien commun}

L'existence de biens collectifs est reconnue dès les fondements de l'économie. Ainsi, Adam Smith révèle, en 1776 dans son ouvrage (Essai sur la Richesse des Nations), l'existence de certains biens qui font partie des "devoirs $d u$ prince», en raison de la dimension collective de la consommation et des difficultés à en assurer le financement privé. Néanmoins, il faut attendre les travaux de Baumol et Bowen (1966) notamment, et la définition de Samuelson (1954), pour que se constitue une véritable théorie des biens communs. Celui-ci propose ainsi une classification des biens selon 2 critères :

- La rivalité ou «non rivalité» : soit la consommation d'un bien par un citoyen empêche la consommation par les autres; soit la consommation du bien par un citoyen n'empêche pas sa consommation par d'autre (du moins tant qu'on n'approche pas la saturation de sa capacité).

- L'exclusion ou la «non exclusion» : soit il est possible d'exclure certains citoyens de la consommation d'un bien; soit il n'est pas possible d'exclure de la consommation pour des raisons techniques et de coûts, les citoyens se situant dans la limite du territoire ou de la juridiction auxquels le bien se réfère.

Lorsque les deux propriétés sont remplies, on parle de «bien collectif pur» ou de bien public, ce qui est en fait assez rare (défense nationale, signalisation des phares, sécurité, signalisation routière, protection nucléaire, inflation, épidémie). Si une seule des deux propriétés est remplie, on parle de «biens collectifs impurs» ou de "biens collectifs mixtes», ou encore de «biens de club» (Buchanan, 1965) pour les biens non rivaux mais excluables, et de «biens communs» pour les biens non rivaux mais non excluables (voir tableau 1). Or, chacune de ces deux propriétés renvoie à une source spécifique d'inefficacité du marché à produire ce type de biens de manière optimale : c'est pourquoi on parle de «défaillance du marché» («market failure»), qui met en échec la «main invisible» de Smith (1776) pour gérer les biens communs.

\begin{tabular}{|l|l|l|}
\hline \multicolumn{3}{|c|}{ TABLEAU 1} \\
\hline \multirow{2}{|c|}{ Typologie des différents types de biens } \\
\hline Rivalité & Excluabilité & Non excluabilité \\
\hline Non Rivalité & $\begin{array}{l}\text { Bien commun ou ressource } \\
\text { à gestion partagée }\end{array}$ \\
\hline
\end{tabular}

Les «biens communs» sont donc ceux dont on ne peut exclure personne de l'usage mais pour lesquels il existe une rivalité dans leur usage. Ces biens sont principalement des ressources naturelles qui s'érodent lorsqu'elles sont consommées à outrance. À ce sujet, Hardin (1968) évoque la tragédie des communs, qui conduit à leur disparition en cas de sur-utilisation. Cette théorie est illustrée par un village d'éleveurs, ou chacun peut faire paître ses animaux dans un pré n'appartenant à personne en particulier. L'usage du pré étant gratuit et sans contrainte, et l'éleveur tirant son revenu de son bétail, l'intérêt de chaque éleveur est donc de conduire ses animaux au pré le plus souvent possible, le plus tôt possible et le plus longtemps possible. Inévitablement, le pré se transforme en champ de boue et devient donc inutilisable par l'ensemble des éleveurs. Dès lors, Hardin (1968) propose deux alternatives pour éviter cette disparition du bien commun :

- La première est une approche «dirigiste», ou autoritaire. Le pré reste le bien commun du village, mais une autorité, disposant d'un pouvoir de sanction, est chargée d'administrer la ressource (le pré) et d'en répartir l'utilisation entre chaque éleveur.

- La deuxième est une approche "capitaliste». Chaque éleveur bénéficie d'un droit de propriété sur une parcelle du pré, et donc se charge d'en gérer la ressource.

Cette théorie montre que la gestion des biens communs requiert une action collective, perpétuellement réévaluée, pour pallier les défaillances du marché. Or, on a longtemps considéré cette théorie comme une preuve de l'intervention obligatoire de la puissance publique pour administrer les biens communs, à l'instar des travaux menés par Musgrave (1959) et Tinbergen (1956), dans le courant de l'école de l'économie publique.

Depuis cette période, d'autres travaux de recherche ont contredit ce postulat. Le théorème de Coase (2005), en introduisant la notion de coûts de transaction, a montré par exemple qu'aucune loi ne permettait de conclure à la supériorité de l'intervention publique, et que la bonne gestion des biens collectifs était plutôt le fruit d'une étude pragmatique, au cas par cas, en comparant les coûts et avantages respectifs (en termes d'efficacité économique et d'intérêt général) de chaque mode de gestion. En clair, pour citer Arrow (1970), «C'est une erreur que de limiter l'action collective à l'action de l'Etat». La gestion de ces biens communs nécessite donc l'émergence de mécanismes collectifs permettant leur gestion. Il 
convient du coup de s'interroger sur les conditions d'existence de tels mécanismes en dehors de la puissance publique.

Les travaux d'Ostrom (2005) complètent la réflexion, en identifiant une régulation autonome des biens communs, par les utilisateurs de la ressources eux-mêmes, à l'image d'une copropriété dans laquelle chacun dispose d'un droit de vote pour prendre une décision collective sur les parties communes. Au-delà des logiques d'intervention de l'Etat et de la régulation sur le marché, Ostrom (2005) a mis en évidence, à partir de multiples études de cas, l'existence de mécanismes d'auto-régulation des biens communs par les consommateurs de la ressource, sous certaines limites : l'inertie de la décision par la recherche d'un consensus mou; l'inaction des passagers clandestins qui ne font pas entendre leur voix lors des délibérations collectives. Dans certaines conditions, le risque est alors d'assister à une dégradation des conditions de la ressource pour satisfaire à la paix sociale dans un cadre d'auto-gestion démocratique.

Ce type de gestion selon Ostrom (2010) est présenté dans différentes situations, suivant 8 principes de conception (tableau 2).

Les travaux d'Ostrom (2010), comme ceux d'Hardin (1968) portent néanmoins sur des ressources naturelles comme l'eau ou les forêts, dont il faut préserver le renouvellement ou répartir au mieux, comme dans le cas de systèmes d'irrigation. Des travaux plus récents mettent en évidence des biens communs qui relèvent d'un construit social ou technologique, c'est-à-dire des biens communs qui ne sont pas préexistants à l'humanité. Ces biens communs émergent alors d'un processus de gestion collective, impliquant de multiples acteurs, qu'il soit institutionnel ou non (Labatut, 2009; Hannachi, 2011). À chaque fois, il s'agit de biens communs qui permettent une création de valeur pour les acteurs (Hannachi et al., 2010), comme la santé des plantes.

Ainsi, sur le marché français, toute variété de fruit développée par une entreprise peut être intégrée dans les programmes de sélection des concurrents. Dès lors, un gène de résistance développé par le semencier A, comme une résistance spécifique aux agresseurs (insectes, champignons, parasites), peut être utilisé par l'ensemble des semenciers B, C, D... à plus ou moins longue échéance. Il n'y a donc pas d'exclusion à l'utilisation de ce gène qui devient un bien commun utilisé par telle ou telle entreprise du secteur d'activité. En effet, chaque entreprise de semence dispose des savoirs techniques lui permettant pour le moins d'intégrer un gène de résistance connue dans son programme de sélection variétale. Ainsi, contrairement aux biens de club que l'on rencontre fortement dans l'agriculture française (Torre, 2002) il est ici impossible d'interdire l'accès à ce bien, pour un nouvel entrant.

Toutefois, dès lors que le contournement d'une résistance par les agresseurs (champignons, parasites, insectes) n'est qu'une question de temps, il y a bien une situation de rivalité pour contrôler ce bien. En effet, l'utilisation en excès d'une résistance spécifique par un, ou plusieurs, semencier entraîne techniquement le contournement rapide de cette résistance génétique et par voie de conséquence, l'impossibilité pour de nouveaux semenciers d'utiliser cette résistance dans leurs programmes de sélection variétale. Ainsi, l'utilisation d'un gène de résistance pour une espèce de fruit n'a plus d'intérêt pour les semenciers, lorsqu'elle est totalement contournée par les prédateurs du fruit. Dès lors, le gène de résistance variétale (et l'innovation technologique pour le réaliser) constitue un bien commun dans l'univers de l'agro-alimentaire, du fait de ses caractéristiques de rivalité et de non excluabilité. Dans cet article nous allons étudier, la manière dont il est possible de gérer durablement le bien commun, c'est-à-dire de prolonger la durée de vie du gène de la résistance pour une espèce de fruit qui n'a pas besoin d'insecticide ou de pesticide : la pomme Ariane ${ }^{\oplus}$.

\section{La méthodologie de la recherche}

Pour répondre à notre problématique, nous réalisons une étude de cas exploratoire suivant la méthode de Yin (1989), afin d'examiner la manière de réguler le bien commun dans l'agriculture durable, à partir de l'innovation technologique dans le domaine de la génétique des plantes, par des mécanismes de régulation intermédiaires aux pouvoirs publics et aux acteurs de marché.

\begin{tabular}{|l|l|}
\hline \multicolumn{2}{|c|}{ TABLEAU 2} \\
Principes d'émergence de biens communs (Orstrom, 2010) \\
$\begin{array}{l}\text { Des limites clairement } \\
\text { définies }\end{array}$ & $\begin{array}{l}\text { Les acteurs possédant des droits d'usage de la ressource sont clairement identifiés, ainsi que les } \\
\text { limites d'appropriation de la ressource commune. }\end{array}$ \\
\hline $\begin{array}{l}\text { Concordance entre règles } \\
\text { d'appropriations et conditions } \\
\text { locales }\end{array}$ & $\begin{array}{l}\text { Les règles qui restreignent l'usage de la ressource sont liées aux conditions locales et aux obligations } \\
\text { en termes de main d'œuvre, de matériels et/ou d'argent et/ou de technologies. }\end{array}$ \\
\hline $\begin{array}{l}\text { Des dispositifs de choix } \\
\text { collectifs }\end{array}$ & Les acteurs concernés par les règles opérationnelles peuvent participer à la modification de celles-ci. \\
\hline $\begin{array}{l}\text { La surveillance } \\
\text { Des sanctions graduelles }\end{array}$ & $\begin{array}{l}\text { Les surveillants, qui examinent les conditions de la ressource et le comportement des appropriateurs, } \\
\text { rendent compte aux appropriateurs. Ils peuvent être désignés parmi les appropriateurs. }\end{array}$ \\
\hline $\begin{array}{l}\text { Les appropriateurs qui transgressent les règles s'exposent à des sanctions graduelles len fonction } \\
\text { de la gravité et du contexte de l'infraction) par les autres appropriateurs ou par des surveillants } \\
\text { travaillant pour leurs compte. }\end{array}$ \\
\hline $\begin{array}{l}\text { Des mécanismes de } \\
\text { résolution des conflits }\end{array}$ & $\begin{array}{l}\text { Les appropriateurs et leurs représentants disposent d'un accès rapide à des arènes de discussion } \\
\text { pour résoudre les conflits entre appropriateurs. }\end{array}$ \\
\hline $\begin{array}{l}\text { Une reconnaissance minimale } \\
\text { des droits d'organisation }\end{array}$ & $\begin{array}{l}\text { Les droits des appropriateurs d'élaborer leurs propres institutions ne sont pas remis en cause par } \\
\text { des autorités gouvernementales externes. }\end{array}$ \\
\hline Des organisations imbriquées & $\begin{array}{l}\text { Les activités d'appropriation, de fourniture, de surveillance, d'application des règles, de résolution } \\
\text { des conflits et de gouvernance sont organisés par de multiples niveaux de régulations imbriquées. }\end{array}$ \\
\hline
\end{tabular}


Cette étude de cas porte notamment sur l'observation d'une collaboration technologique publique-privée entre un laboratoire de recherche rattaché à l'INRA (Institut National de Recherche Agronomique), et un consortium de petites entreprises agricoles pour le développement et la commercialisation d'une nouvelle variété de pomme résistante aux agressions d'un champignon : la pomme Ariane ${ }^{\circledast}$.

Sur le plan empirique, l'étude de cas exploratoire menée sur la pomme Ariane ${ }^{\circledast}$ doit nous aider à comprendre les modalités de régulation d'un bien commun comme l'innovation génétique dans une filière agro-alimentaire : soit par la régulation publique du laboratoire de l'INRA suivant des critères d'intérêt général pour la filière (Coléno et Ould Ahmed 2009); soit par la régulation privée des acteurs de la filière avec la mise en œuvre de stratégie collective au sens d'Astley et Fonbrum (1983), afin d'exploiter cette innovation suivant des critères purement économiques (Ould Ahmed 2008); soit par la combinaison des deux modes de régulation public et privé afin de concilier simultanément la dimension environnementale et économique.

Pour réaliser cette étude de cas nous avons collecté des données primaires issues d'entretiens semi directifs avec plusieurs dirigeants des PME partenaires, complétés par des entretiens avec des chercheurs du laboratoire de l'INRA, jusqu'à saturation des données. Afin de vérifier la validité des données nous avons recoupé les données primaires avec des données secondaires (Jick, 1979) auprès de dirigeants de l'INRA extérieurs au projet, mais disposant d'une visibilité institutionnelle afin de mieux comprendre les modalités conventionnelles de partenariat

\begin{tabular}{|l|l|}
\hline \multicolumn{2}{|c|}{ TABLEAU 3 } \\
\multicolumn{2}{|c|}{ Liste des entretiens issus de } \\
\hline Fonction de l'interviewé & Entreprise ou organisme \\
\hline Gérant & Entreprise pépiniériste \\
\hline Co-gérant & $\begin{array}{l}\text { Joint-venture pépiniéristes } \\
\text { (NOVADI) }\end{array}$ \\
\hline Gérant & Entreprise de mise en marché \\
\hline Directeur général & Club variété \\
\hline $\begin{array}{l}\text { Responsable du programme } \\
\text { de sélection Ariane }{ }^{\circledR}\end{array}$ & INRA \\
\hline Chef de département & INRA \\
\hline Chef de département & INRA \\
\hline
\end{tabular}

public-privé dans l'agro-alimentaire (Ould Ahmed, 2008). Le tableau 3 ci-dessous présente l'échantillon de contacts dans l'enquête empirique menée sur la période 2007-2010.

\section{Une variété gérée en bien commun : la pomme Ariane ${ }^{\circledR}$}

\section{La pomme Ariane}

La pomme Ariane ${ }^{\circledast}$ est une variété de pomme développée par l'INRA et plusieurs obtenteurs privés. Le développement de cette variété repose sur un programme de sélection de 50 ans développé par l'INRA pour lutter contre la tavelure et une recherche de variété de pomme répondant à des caractéristiques gustatives particulières (pomme juteuse et sucrée). Cette variété de pomme vise un marché de niche à forte valeur ajouté occupé principalement par la variété pink lady ${ }^{\circledR}$. Depuis 2008 la production de pommes ariane $e^{\varpi}$ croit régulièrement passant de 14000 tonnes produites en 2008 à 24000 tonnes en 2013. Elle occupe aujourd'hui 1,5\% du marché français de la pomme (voir tableau 4).

L'atout majeur d'Ariane réside dans sa résistante naturelle à la tavelure, causée par le champignon Venturia inaequalis, qui est la maladie la plus préjudiciable du pommier et dont la protection nécessite de nombreux traitements fongicides chaque année. Cette résistance permet de réduire le nombre de traitements.

«Ces applications fongicides régulières agissent négativement sur la faune auxiliaire et peuvent favoriser le développement de certains ravageurs. De plus, des problèmes de résistance à certaines matières actives apparaissent régulièrement et de nombreuses situations d'échecs de protection contre la tavelure sont apparues ces dernières années dans l'ensemble du verger français» (François Laurens, INRA GenHort)

La majorité des variétés résistantes actuellement disponibles portent le même gène de résistance majeure $(V f)$ qui a été contourné dans les années 1985-1990. Les premiers contournements d'Ariane ont, quant à eux, été observés dans les vergers de l'INRA dès 2004. Il y a eu des contournements de la résistance dans les vergers des arboriculteurs qui exploitent Ariane. Théoriquement, ce contournement peut être généralisé, ce qui risque de faire perdre à la variété une de ses caractéristiques les plus intéressantes. Pour limiter ces contournements, l'INRA a proposé un ensemble de dispositifs de régulation pouvant prolonger la durée de vie de la résistance variétale.

\section{La régulation publique du bien commun : l'innovation de la pomme Ariane $^{\infty}$}

Une cinquantaine d'équipes de recherche à travers le monde développe aujourd'hui des programmes de recherche dont l'objectif est la création de nouvelles variétés de pommes. Ils sont répartis sur tout les continents mais de façon majoritaire en Europe où l'on compte en moyenne un programme par pays. La France est très active dans ce domaine, dans la mesure où elle occupe la place du deuxième producteur européen de pommes derrière l'Italie. En dehors du programme public mené par l'INRA, plusieurs laboratoires privés sont également impliqués dans la création variétale. L'objectif commun partagé par toutes les équipes de recherche à travers le monde consiste à créer de nouvelles variétés, combinant d'excellentes qualités de goût (attrait visuel et qualité gustative), de longue conservation, et de résistance aux bioagresseurs.

Or, la tavelure, causée par le champignon Venturia inaequalis est la principale maladie du pommier. Toutes les grandes variétés commerciales y sont sensibles voire extrêmement sensibles. La tavelure justifie en moyenne la moitié des traitements phytosanitaires du verger. Dans cette mesure, l'innovation sur les variétés résistantes de pomme se concentre depuis de nombreuses années sur la protection contre la tavelure.

Pour répondre à cet enjeu de protection, Ariane ${ }^{\circledast}$ est née en France, dans le cadre original et novateur d'une démarche 
d'innovation publique-privée. Cette variété est issue d'un croisement initial, réalisé aux Etats-Unis, entre une espèce sauvage de pommier (Malus floribunda) qui porte deux gènes de résistance à la tavelure, mais donnant des fruits minuscules, et une variété de pommier possédant des qualités agronomiques et gustatives satisfaisantes. Plusieurs croisements successifs ont ensuite été réalisés pour sélectionner des gènes de très bonne qualité gustative et porteurs de résistance, à l'issue d'une trentaine d'années de recherches menées par l'INRA en collaboration avec des pépiniéristes. Elle est la première pomme de grande consommation à posséder une résistance naturelle à la tavelure du pommier permettant ainsi une réduction, de l'ordre de $50 \%$, du nombre de traitements phytosanitaires pratiqués dans les vergers. Elle est considérée comme l'avancée technique la plus importante de ces 20 dernières années dans le monde de l'arboriculture.

Le programme de recherche concernant une telle pomme a démarré dans les années 50 sous la houlette du laboratoire public GenHort de l'INRA, pour aboutir à un résultat final en 1979. Sous le nom de code X 6407, la variété hybride Ariane ${ }^{\circledast}$ découverte en 1979, compte une trentaine d'ascendants dont la Golden delicious, la Starking Simpson, la Jonathan, la Prima et l'hybride initial porteur du gène qui confère la résistance à la tavelure. À partir de ce résultat clinique en laboratoire, différentes expérimentations ont ensuite été nécessaires pour l'évaluation in situ. Il aura fallu vingt années d'essais en serre au sein de l'INRA, puis en vergers expérimentaux avant qu'Ariane ${ }^{\bowtie}$ ne soit inscrite officiellement au catalogue des variétés de pomme commercialisable en 2002. Durant cette longue période d'expérimentation in situ, le laboratoire GenHort de l'INRA a été conduit à restructurer ses collaborations avec les pépiniéristes, non pas comme un prestataire de service, mais comme partenaire dans le cadre d'un réseau public-privé.

À partir de 1997, le laboratoire de recherche de l'INRA change en effet sa politique relationnelle avec les pépiniéristes afin de diminuer le coût de ces collaborations bilatérales et de réaliser des économies d'échelle dans l'expérimentation par des collaborations multilatérales. Il exige des pépiniéristes, rivaux sur le plan commercial, qu'ils se regroupent dans une seule et même entreprise, afin de disposer d'un seul interlocuteur, de manière à rationaliser les coûts.

Face à cette exigence, les pépiniéristes se regroupent dans une filiale commune, une joint venture : NOVADI. La joint-venture NOVADI a pour but d'assurer le développement et la commercialisation des variétés résistantes à la tavelure développée par l'INRA avec certains pépiniéristes (Garmendia et al., 2009). Ce

\begin{tabular}{|c|c|c|c|c|c|c|c|c|c|c|c|c|}
\hline \multirow[b]{2}{*}{ Variétés } & \multicolumn{12}{|c|}{ x $1000 \mathrm{~T}$} \\
\hline & 2003 & 2004 & 2005 & 2006 & 2007 & 2008 & 2009 & 2010 & 2011 & 2012 & 2013 & $2013 /$ moy.5 ans \\
\hline Golden & 680 & 658 & 660 & 581 & 616 & 543 & 605 & 564 & 581 & 336 & 507 & $-4 \%$ \\
\hline Gala & 289 & 283 & 305 & 272 & 290 & 266 & 261 & 246 & 269 & 226 & 270 & $6 \%$ \\
\hline Granny & 188 & 180 & 185 & 173 & 174 & 172 & 186 & 183 & 210 & 147 & 169 & $-6 \%$ \\
\hline Pink Lady & 50 & 54 & 65 & 66 & 73 & 69 & 82 & 89 & 92 & 78 & 102 & $24 \%$ \\
\hline Braeburn & 155 & 154 & 167 & 141 & 144 & 115 & 118 & 94 & 102 & 61 & 94 & $-4 \%$ \\
\hline Rouges & 104 & 109 & 99 & 84 & 75 & 61 & 74 & 67 & 82 & 47 & 59 & $-11 \%$ \\
\hline Fuji & 33 & 32 & 42 & 41 & 49 & 49 & 68 & 62 & 63 & 44 & 65 & $14 \%$ \\
\hline Belchard & 19 & 21 & 24 & 27 & 33 & 34 & 38 & 37 & 44 & 37 & 52 & $37 \%$ \\
\hline Canada & 41 & 44 & 43 & 40 & 42 & 39 & 40 & 41 & 46 & 23 & 46 & $22 \%$ \\
\hline Ariane & & & & & & 14 & 18 & 21 & 21 & 18 & 24 & $30 \%$ \\
\hline Elstar & 34 & 29 & 31 & 26 & 24 & 17 & 18 & 15 & 17 & 14 & 22 & $36 \%$ \\
\hline Honey Crunch & & & & & & & 5 & 12 & 13 & 13 & 14 & \\
\hline Jazz & & & & & & & 13 & 13 & 16 & 12 & 15 & \\
\hline R.Reinettes & 15 & 20 & 20 & 17 & 14 & 15 & 13 & 13 & 14 & 11 & 12 & $-9 \%$ \\
\hline Jonagold & 38 & 34 & 34 & 25 & 28 & 22 & 20 & 15 & 14 & 6 & 23 & $49 \%$ \\
\hline Tentation & & & 9 & 10 & 9 & 9 & 8 & 8 & 7 & 6 & 7 & $-8 \%$ \\
\hline Joya & & & & & & & & 8 & 7 & 6 & 8 & \\
\hline Idared & 17 & 20 & 17 & 16 & 11 & 9 & 7 & 6 & 6 & 4 & 10 & $56 \%$ \\
\hline Boskoop & & 11 & 10 & 9 & 11 & 10 & 9 & 6 & 7 & 4 & 10 & $39 \%$ \\
\hline Caméo & & & 7 & 9 & 5 & 7 & 6 & 3 & 2 & 1 & 2 & $-47 \%$ \\
\hline Choupette & & & & & & & 1 & 3 & 3 & 2 & 3 & \\
\hline Autres & 65 & 60 & 52 & 48 & 78 & 77 & 61 & 73 & 85 & 73 & 62 & $-16 \%$ \\
\hline TOTAL & 1728 & 1709 & 1770 & 1585 & 1676 & 1528 & 1651 & 1579 & 1701 & 1169 & 1576 & $3 \%$ \\
\hline
\end{tabular}


regroupement au sein de NOVADI permet aux pépiniéristes d'améliorer les collaborations avec le laboratoire public, dans le but aussi d'optimiser sur toute la filière l'implantation des vergers Ariane ${ }^{\varpi}$, afin de minimiser les risques de transfert de maladie, d'un verger à l'autre.

Une telle association en joint-venture entre PME concurrentes est particulièrement rare. En effet, la R\&D étant destinée à créer des produits délivrant un avantage compétitif, elle fait rarement l'objet d'associations entre concurrents. De telles associations s'observent toutefois dans d'autres secteurs d'activités comme dans l'industrie automobile japonaise par exemple. Dans cette industrie, le rapprochement entre concurrents s'effectue par la volonté d'un même client de voir l'ensemble de ses fournisseurs collaborer pour tirer parti de l'intelligence collaborative, comme dans le cas d'un club de fournisseurs (Sako, 1996). Par contre, en dehors du secteur des biotechnologies, et plus largement à l'égard des PME innovantes, il est rare que des laboratoires publics comme l'INRA jouent un rôle actif pour fédérer les collaborations entre concurrents privés (Dalpé, 2003).

Le réseau public-privé constitué par l'INRA et le groupement de pépiniéristes NOVADI, a ainsi pour objectif de mettre en commun les capacités d'innovation des différents pépiniéristes et de l'INRA. Ce réseau mutualise les collections de variétés et les compétences de création variétale des différentes entreprises pour les partager avec le laboratoire de l'INRA. Le laboratoire de l'INRA joue un rôle fédérateur dans ce réseau, du fait des compétences et des moyens en sélection dont il dispose, et qui sont supérieurs à ceux de chacun des autres partenaires privés. Dans ce contexte, l'innovation publique-privée au sein de la filière de la pomme peut être considérée comme un bien commun.

En effet, dans la typologie des innovations technologiques, plusieurs situations se distinguent. L'innovation fermée, de type incrémentale ou radicale, a pour caractéristique de conférer des droits exclusifs de propriété de l'innovation à une entreprise isolée ou à un groupement d'entreprises privées liées par contrat. Dans ce contexte, l'innovation prend le statut d'un bien privé, conférant à son propriétaire un avantage concurrentiel pour exclure les concurrents du marché. En revanche, lorsque l'innovation est de type ouverte, en nécessitant des collaborations multiples, elle prend la forme : soit d'un bien de club à l'intérieur d'un groupement d'entreprises privées qui détiennent ainsi un avantage concurrentiel collectif (Loilier et Tellier 1999); soit la forme d'un bien commun dans le cadre d'un partenariat public-privé lorsque l'innovation transforme radicalement la nature d'un secteur d'activité, en définissant un nouveau standard de production et de consommation. C'est justement le cas de l'innovation sur la pomme Ariane ${ }^{\varpi}$, comme le résume le tableau 5 ci-dessous.

Dans le cas Ariane ${ }^{\varpi}$, le laboratoire public INRA assume un rôle de régulation important, en raison de sa neutralité par rapport aux rivalités commerciales sous-jacentes des partenaires privés, concurrents. De ce point de vue, l'INRA reste neutre, indépendant, soucieux du bien commun de l'innovation technologique pour la filière. Néanmoins, l'action de l'INRA a ses limites, comme tout acteur public visant à réguler un marché, selon l'un des responsables de l'INRA du département de génétique et d'amélioration des plantes :

"L'INRA génère des connaissances, ensuite c'est aux politiques et aux acteurs de terrain d'intervenir sur l'exploitation de ces connaissances, pour développer des schémas de production dans un sens ou dans un autre. On peut défendre une agriculture durable nécessitant peu de pesticides, mais on ne peut pas aller plus loin.»

Au-delà de l'activité de R\&D pour produire l'innovation, il s'agit maintenant de comprendre comment il est possible de préserver les intérêts communs dans la filière de la pomme, alors que de multiples entreprises privées cherchent à défendre leurs intérêts commerciaux.

\section{La régulation privée du bien commun : la filière de la pomme} Ariane ${ }^{\oplus}$

Contrairement aux grandes cultures de céréales, où l'agriculteur change de production (et de variété) tout les ans, la production de pommes est une production pérenne. L'implantation d'une variété est une décision qui engage les arboriculteurs à long terme. L'implantation d'Ariane ${ }^{\circledast}$ sur plus de 800 hectares, oblige l'ensemble de la filière et pas uniquement les pépiniéristes, à produire et à commercialiser cette variété durant une dizaine d'années au moins. En raison de cette dépendance mutuelle entre l'INRA, les arboriculteurs, les pépiniéristes, le réseau public-privé à l'origine de l'innovation sur la pomme Ariane ${ }^{\oplus}$, commence à s'étendre en 2002 , par capillarité à tout les acteurs de la filière.

Les 17 pépiniéristes regroupés au sein de NOVADI décident ainsi de s'associer avec d'autres entreprises complémentaires sur la chaîne de valeur parmi lesquelles les organisations de producteurs $(\mathrm{OP})$ et les metteurs en marché :

- Les organisations de producteurs (OP) sont des groupements d'arboriculteurs. Ils organisent la production de ces derniers et permettent le regroupement des achats d'intrants. Ils assurent en outre la commercialisation des productions de leurs adhérents. Les organisations de producteurs jouent un rôle moteur dans le secteur en permettant la concentration

\begin{tabular}{|l|l|l|}
\hline \multicolumn{3}{|c|}{ TABLEAU 5} \\
& \multicolumn{1}{|c|}{ Typologie des innovations } \\
\hline & Innovation fermée & Innovation ouverte \\
\hline $\begin{array}{l}\text { Innovation incrémentale } \\
\text { (de process) }\end{array}$ & $\begin{array}{l}\text { R\&D interne aux grandes entreprises } \\
\text { Innovation = Bien privé }\end{array}$ & $\begin{array}{l}\text { Association de PME (parc technologique) } \\
\text { Innovation = Bien de club }\end{array}$ \\
\hline $\begin{array}{l}\text { Innovation de rupture } \\
\text { (de saut technologique) }\end{array}$ & $\begin{array}{l}\text { Partenariat PME -Grande entreprise } \\
\text { Innovation }=\text { Bien privé }\end{array}$ & $\begin{array}{l}\text { Partenariat public privé PME - organisme public } \\
\text { Innovation }=\text { Bien commun (cas Ariane }{ }^{\circledR} \text { ) }\end{array}$ \\
\hline
\end{tabular}


de l'offre et l'amélioration des techniques de production et des méthodes de commercialisation. Les Organisations des Producteurs sont pour la plupart des coopératives ou des arboriculteurs individuels réunis pour commercialiser leur production ensemble. L'adhésion au réseau est la seule possibilité pour ces groupements de producteurs de pouvoir disposer de jeune plans d'Ariane ${ }^{\circledast}$ pour leurs adhérents.

- Les metteurs en marché sont des agences assurant la distribution et la commercialisation de la production des OP. Ils constituent un passage obligé pour vendre la production des arboriculteurs. Le metteur en marché garantit le stockage et l'acheminement des produits dans les meilleures conditions, afin d'assurer une qualité optimale des produits. Installés dans les bassins de production, ils sont d'importants employeurs de main-d'œuvre, même si beaucoup d'opérations, notamment au niveau du tri et du calibrage, sont aujourd'hui mécanisées et informatisées. Les organismes de producteurs s'engagent à ne commercialiser la production de leurs adhérents que via des metteurs en marchés eux même membre du réseau, renforçant en cela la logique d'exclusivité dans l'appartenance au réseau Ariane ${ }^{\circledast}$. L'enjeu commercial est important, car la résistance à la tavelure constitue un avantage pour les consommateurs, mis en avant à travers le slogan : «Quand on soigne bien la nature elle nous donne le meilleur». Cette différenciation sur l'image de marque permet en outre de nouer des transactions privilégiées en bout de chaîne, avec la grande distribution comme Carrefour ou Auchan.

L'association entre tout ces acteurs se déroule dans une structure commune POMALIA, sous statut SAS société en action simplifiée ${ }^{1}$. La structure POMALIA, dont le nom provient de l'union de deux mots : Pomme et alliance, regroupant NOVADI (17 pépiniéristes membres), mais également 38 Organisations de Producteurs (OP) et 6 metteurs en marché. Chacune de ces familles professionnelles contrôle un tiers des actions de la société.

La vocation de cette structure est d'organiser la production et la commercialisation d'une gamme de variétés de pommes résistantes à la tavelure : la pomme Ariane ${ }^{\varpi}$ Le regroupement dans POMALIA des pépiniéristes, d'OP et de metteurs en marché, représentant les trois maillons de création de valeur (semences, production, distribution), permet de mettre en place une véritable structure de concertation et de décision dans la filière.

POMALIA coordonne les plantations (niveau production) et harmonise la stratégie marketing (niveau distribution). Le regroupement de tout les acteurs de la filière de la pomme au sein d'une structure commune POMALIA permet de :

- Mutualiser les efforts de communication liés à l'introduction d'une nouvelle variété. Un tel effort est en effet important au regard de la taille économique des acteurs concernés. La création d'une structure de gouvernance commune, entre les trois catégories d'acteurs (pépiniéristes, groupements de producteurs et metteurs en marché) permet de répartir les coûts.

- Maitriser l'image et la qualité du produit en sélectionnant les producteurs les plus à même d'assurer une production de qualité. La mise en place d'une structure de gouvernance collégiale à travers POMALIA, permet en effet de sélectionner les membres à l'entrée.

- Assurer un développement optimisé de la variété. L'objectif pour l'ensemble des acteurs, y compris GenHort de l'INRA, qui touche des royalties sur la production de pomme, consiste à optimiser le développement de la production en fonction de l'accroissement de la demande. Ainsi, les marges des différents acteurs, semenciers, producteurs, distributeurs, sont préservées, ce qui n'est pas le cas pour d'autres variétés de pomme, comme la Golden, où la grande distribution capte la majeure partie de la valeur ajoutée au détriment des agriculteurs. Un nouveau producteur peut ainsi adhérer au groupement dès lors qu'il existe un marché permettant de répondre à l'augmentation de production, sans perte de valeur pour le groupement.

- Assurer la durabilité de la résistance de la variété. Cet objectif repose sur la promotion de bonnes pratiques dans l'exploitation des vergers, en lien avec les travaux de recherche de l'INRA. Ainsi, les travaux de l'INRA sur ce sujet sont régulièrement présentés aux membres de POMALIA.

La gouvernance du développement de la variété Ariane ${ }^{\varpi}$ répond ainsi aux principes de gouvernance des biens communs mis en évidence par Orstrom (2005) comme le montre le tableau 6. Ainsi, l'intervention d'un tiers reconnu comme légitime par l'ensemble des acteurs peut permettre l'émergence d'une communauté d'acteurs regroupés autour de la gestion durable d'un bien.

\section{La régulation publique-privée du bien commun : le réseau administré}

La structure de gouvernance de POMALIA regroupe 17 pépiniéristes, 38 Organisations de Producteurs (OP) et 6 metteurs en marché. Chacune de ces familles professionnelles réunies en collège, contrôle un tiers des actions de la société. Cela signifie que chaque collège (pépiniériste, $\mathrm{OP}$ et metteurs en marchés) dispose de 3 représentants dans le comité de direction sur un total de 9 représentants. Les décisions sont donc issues d'un consensus entre les représentants des trois collèges.

Pour prendre une décision qui s'applique à tout les acteurs, il est en effet impératif de disposer de 7 voix sur les 9, ce qui élimine la possibilité que deux collèges s'unissent contre l'autre. C'est un point important, car cela signifie que la répartition de la valeur économique créée conjointement dans la filière, ne peut pas être captée par un maillon (semenciers, producteurs, distributeurs) au détriment des autres.

C'est une manière de répondre à une exigence de développement durable cette fois-ci sur le plan économique en considérant la filière de la pomme comme un écosystème où chaque acteur dépend des autres pour sa propre survie de long terme. Dans le domaine de l'agroalimentaire, cette situation n'est pas courante.

La plupart du temps, la négociation entre les fournisseurs, les producteurs et les distributeurs ne parvient pas toujours à s'équilibrer suivant les filières (Desquilbet et Guyomard 2002). Par exemple, d'après le centre d'information sur les viandes, en 2010, en France, le prix au kilo de viande bovine entre le producteur et le consommateur est multiplié par 8,5 au bénéfice principalement des intermédiaires et de la grande distribution,

1. SAS : société par action simplifiée qui est à la fois une société de capitaux et de personnes, une société mixte à l'image d'une société anonyme assortie d'un pacte d'actionnaires. 


\begin{tabular}{|c|c|c|}
\hline \multicolumn{3}{|c|}{$\begin{array}{l}\text { TABLEAU } 6 \\
\text { Principes de gestion en bien commun de la pomme Ariane }{ }^{\circledR}\end{array}$} \\
\hline $\begin{array}{l}\text { Des limites clairement } \\
\text { définies }\end{array}$ & $\begin{array}{l}\text { Les acteurs possédant des droits d'usage de la ressource sont } \\
\text { clairement identifiés, ainsi que les limites d'appropriation de la } \\
\text { ressource commune. }\end{array}$ & $\begin{array}{l}\text { Seul les membres de pomalia peuvent } \\
\text { commercialiser la variété Ariane. }\end{array}$ \\
\hline $\begin{array}{l}\text { Concordance entre } \\
\text { règles d'appropriations } \\
\text { et conditions locales }\end{array}$ & $\begin{array}{l}\text { Les règles qui restreignent l'usage de la ressource sont liées aux } \\
\text { conditions locales et aux obligations en termes de main d'œuvre, } \\
\text { de matériels et/ou d'argent et/ou de technologies. }\end{array}$ & $\begin{array}{l}\text { Le développement d'Ariane est lié à } \\
\text { l'existence d'un marché suffisant et à } \\
\text { des pratiques. }\end{array}$ \\
\hline $\begin{array}{l}\text { Des dispositifs de choix } \\
\text { collectifs }\end{array}$ & $\begin{array}{l}\text { Les acteurs concernés par les règles opérationnelles peuvent } \\
\text { participer à la modification de celles-ci. }\end{array}$ & $\begin{array}{l}\text { Les règles opérationnelles sont } \\
\text { discutés lors des } A G \text { de Pomalia. }\end{array}$ \\
\hline La surveillance & $\begin{array}{l}\text { Les surveillants, qui examinent les conditions de la ressource et le } \\
\text { comportement des appropriateurs, rendent compte aux } \\
\text { appropriateurs. Ils peuvent être désignés parmi les appropriateurs. }\end{array}$ & $\begin{array}{l}\text { L'inra et les membres du réseaux } \\
\text { assurent un suivi du contournement } \\
\text { de la résistance et en informent les } \\
\text { membres du réseau. }\end{array}$ \\
\hline $\begin{array}{l}\text { Des sanctions } \\
\text { graduelles }\end{array}$ & $\begin{array}{l}\text { Les appropriateurs qui transgressent les règles s'exposent à des } \\
\text { sanctions graduelles (en fonction de la gravité et du contexte de } \\
\text { l'infraction) par les autres appropriateurs ou par des surveillants } \\
\text { travaillant pour leurs compte. }\end{array}$ & $\begin{array}{l}\text { Le non-respect des règles de bonne } \\
\text { pratiques eput donner lieu à des } \\
\text { sanctions prise au niveau du } \\
\text { groupement de producteur. }\end{array}$ \\
\hline $\begin{array}{l}\text { Des mécanismes de } \\
\text { résolution des conflits }\end{array}$ & $\begin{array}{l}\text { Les appropriateurs et leurs représentants disposent d'un accès } \\
\text { rapide à des arènes de discussion pour résoudre les conflits entre } \\
\text { appropriateurs. }\end{array}$ & $\begin{array}{l}\text { Discussion au sein des différents } \\
\text { collèges puis, si nécessaire au sein de } \\
\text { pomalia. }\end{array}$ \\
\hline $\begin{array}{l}\text { Une reconnaissance } \\
\text { minimale des droits } \\
\text { d'organisation }\end{array}$ & $\begin{array}{l}\text { Les droits des appropriateurs d'élaborer leurs propres institutions } \\
\text { ne sont pas remis en cause par des autorités gouvernementales } \\
\text { externes. }\end{array}$ & $\begin{array}{l}\text { L'organisation est constituée de } \\
\text { plusieurs groupements de de droit } \\
\text { privé. }\end{array}$ \\
\hline $\begin{array}{l}\text { Des organisations } \\
\text { imbriquées }\end{array}$ & $\begin{array}{l}\text { Les activités d'appropriation, de fourniture, de surveillance, } \\
\text { d'application des règles, de résolution des conflits et de gouvernance } \\
\text { sont organisés par de multiples niveaux de régulations imbriquées. }\end{array}$ & $\begin{array}{l}\text { Structuration en collège différents } \\
\text { regroupés au sein de POMALIA. }\end{array}$ \\
\hline
\end{tabular}

et donc au détriment du producteur local. De même dans la production de lait et dans la filière des fruits et légumes, les agriculteurs français souffrent d'une captation des marges par la grande distribution.

Par rapport à l'organisation de la filière de la pomme Ariane ${ }^{\circledast}$, l'INRA occupe une position particulière. Il n'est pas membre de la structure de gouvernance POMALIA et ne prend pas part aux décisions, mais son rôle d'innovateur lui confère une position stratégique au sein du réseau. La sélection variétale sur le plan technologique, continue d'être assurée par l'INRA. L'INRA assure en outre le suivi des contournements de résistance à la tavelure et met en œuvre des recherches pour limiter ces contournements. Le responsable du programme de sélection pomme Ariane ${ }^{\circledast}$ de l'INRA participe comme invité, aux assemblées générales de POMALIA. Ainsi, l'INRA répond à l'une de ses missions

D’une manière plus générale, la structure POMALIA est caractéristique d'un réseau administré (Assens, 2013). Plus précisément, la collaboration entre les membres de POMALIA relève de règles institutionnelles : une charte des droits et des devoirs dans l'adhésion au réseau. Une structure de gouvernance animée par des représentants élus parmi les membres du réseau, est chargée de faire évoluer ces règles et de vérifier leur application auprès de tout les adhérents.

Cette structure de gouvernance incarne la personnalité morale du réseau. Elle engage la responsabilité juridique du réseau et dispose de la légitimité pour résoudre les blocages, arbitrer les conflits, définir les axes de rayonnement. En conséquence, le réseau administré correspond à une véritable co-entreprise basée sur l'affectio societatis: l'intention des membres d'être considérés comme des égaux dans la structure de gouvernance (Hatchuel et Segrestin 2007).

Ce modèle est dominant dans l'économie sociale et solidaire, dans le secteur associatif; dans le domaine des banques mutualistes dont le capital social est réparti auprès des clients-sociétaires; auprès des mutuelles de santé où la solidarité s'exerce dans les cotisations entre malades et bien portants. La structure de gouvernance en réseau administré est particulièrement adaptée pour réguler des enjeux de bien commun, comme l'exploitation d'une innovation technologique pour le développement durable d'une filière. Le tableau ci dessous en résume les principaux éléments.

En d'autres termes, la stratégie de mise en réseau de l'acteur public avec les acteurs privés à différents stades de valorisation, permet de créer une communauté de destin favorable au développement commercial de la variété de la pomme Ariane ${ }^{\varpi}$ selon la co-gérante de NOVADI, membre du comité de direction de POMALIA :

"Les différents actionnaires de l'entreprise POMALIA sont réunis pour valoriser la variété Ariane ${ }^{\circledast}$, ce faisant, ils sont liés par un destin commun car le succès de l'entreprise et de la variété est un succès pour tout le monde et vice versa. De notre part, on veille à renforcer cette relation de dépendance afin que tout le monde ait une plus value à travers l'exploitation de la licence exclusive que nous a accordée l'INRA...Nos relations avec l'INRA sont toujours très importantes. Nous avons en effet plusieurs contrats de recherche avec eux. En particulier pour continuer de travailler sur Ariane $»$. 


\begin{tabular}{|c|c|}
\hline & Réseau public-privé / Gouvernance administrée (structure POMALIA] \\
\hline $\begin{array}{l}\text { Pacte } \\
\text { fondateur } \\
\text { du réseau }\end{array}$ & $\begin{array}{l}\text { - Solidarité entre partenaires publics et privés fondée sur l'affectio societatis : l'égalité de traitement avec une charte } \\
\text { des droits et des devoirs. } \\
\text { - Logique de cooptation entre membres fondateurs et nouveaux membres pour entrer dans le réseau. } \\
\text { - L'innovation technologique Ariane }{ }^{\circledR} \text { (bien commun) justifie la création du réseau pour valoriser les compétences } \\
\text { publiques et privées. }\end{array}$ \\
\hline $\begin{array}{l}\text { Architecture } \\
\text { du réseau }\end{array}$ & $\begin{array}{l}\text { - Maillage par des relais têtes de réseau constitués par les représentants des trois collèges professionnels : } \\
\text { pépiniéristes, arboriculteurs, distributeurs. } \\
\text { - L'innovation technologique Ariane }{ }^{\circledR} \text { (bien commun) fédère l’appartenance au réseau. } \\
\text { - Les frontières d’appartenance au réseau conditionne l'utilisation de la variété Ariane }{ }^{\circledR} \text {. }\end{array}$ \\
\hline $\begin{array}{l}\text { Règle du jeu } \\
\text { relationnel }\end{array}$ & $\begin{array}{l}\text { - L'innovation technologique Ariane }{ }^{\circledR} \text { (bien commun) est régulée par la recherche de consensus entre pairs. } \\
\text { - Gouvernance par des représentants élus de manière « démocratique ». } \\
\text { - Risque de pouvoir grandissant de la technostructure si le réseau se développe, et donc de perte de la représentatitivité } \\
\text { démocratique. }\end{array}$ \\
\hline
\end{tabular}

\section{Conclusion}

L'histoire de la pomme Ariane ${ }^{\bowtie}$, variété résistante à la tavelure, est riche d'enseignements pour comprendre la manière de répondre à la question du développement durable dans l'agriculture, en considérant que cette question relève de la gestion d'un bien commun, impliquant à la fois des acteurs publics et privés. Dans le prolongement des travaux d'Orstrom (2010), le cas d'Ariane met ainsi en évidence, que des communautés d'acteurs sont en mesure de s'organiser pour gérer un bien commun, afin d'éviter que ne disparaissent des ressources utiles pour la survie de cette communauté. Nous montrons, en outre, à travers ce cas que des modes de gestion collectifs peuvent préexister à l'émergence d'un bien commun, comme l'a montré Labatut (2009) dans ses travaux sur les races animales. La préexistence de ces modalités de gestion est permise par l'action d'un tiers de confiance (Geindre 2005), dont la neutralité induit la bonne gestion collective (Hannachi et Coléno, 2015). Dans ces conditions, il est toutefois nécessaire que le bien commun procure un avantage économique durable aux acteurs impliqués dans le processus de gestion.

Dans cette recherche, nous considérons que le bien commun doit donc être administré simultanément par les pouvoirs publics et par les acteurs privés, pour éviter à la fois les risques de gestion à court terme sur des bases uniquement politiques, ou les risques de tensions individuelles sur des bases uniquement économiques. Pour atteindre cet objectif, la régulation du bien commun doit relever d'une mise en réseau des acteurs publics et privés le long de la chaîne de valeur de la filière agricole.

Plus précisément, dans la chaîne de valeur de la filière de la pomme Ariane ${ }^{\oplus}$, l'acteur public INRA, agit en amont de la création de valeur, puisqu'il structure l'offre agro-alimentaire, à partir d'une innovation technologique qu'il a mise au point pour répondre aux attentes sur le marché. Cette innovation vise à concilier les intérêts économiques des acteurs de la filière en leur permettant de conserver des rendements de production importants, tout en préservant la biodiversité et les ressources naturelles sur les terres cultivables par la réduction des pesticides. De ce point de vue, l'innovation technologique portant sur la gestion des variétés résistantes relève de la logique de bien commun dans la filière agro-alimentaire, car elle conditionne à long terme la survie économique de tout les acteurs.
Autrement-dit, imaginer une agriculture durable, capable de préserver les ressources naturelles de la terre tout en nourrissant une population mondiale en expansion, ne peut donc se concevoir sans la préservation des équilibres entre l'écosystème (les acteurs de la filière) et le biosystème (environnement naturel). Un acteur public comme l'INRA peut jouer un rôle fondamental dans ce domaine, non pas en se substituant aux acteurs privés, mais en servant de trait d'union à l'intérieur d'un réseau public-privé, de manière à garantir le respect du bien commun, à mi-chemin entre l'intérêt général du pays et l'intérêt particulier d'une filière économique. Ceci pose toutefois la question des missions assumées par l'INRA, institut de recherche publique, dont on pourrait penser qu'elles se cantonnent à la production de connaissance, comme les autres instituts de recherches français (CNRS, INSERM,...). Contrairement à ceux-ci la valorisation des recherches de l'INRA fait explicitement parti de ses missions «l'INRA... a pour mission... $5^{\circ}$ : de participer à la valorisation de ses recherches et de son savoir-faire» (Article R831-1 du code rural, version en vigueur jusqu'au 26 novembre 2015). Les expériences du type de celles que nous avons analysé ici ont en outre amené à renforcer et préciser cette mission de l'INRA. Ainsi, l'article R831-1 du code rural précise désormais : «l'INRA... Contribue, par la valorisation de ses compétences, de ses savoir-faire et des résultats de la recherche, à la conception d'innovations technologiques et sociales"

Au delà des apports dans la connaissance, cette recherche comporte un certain nombre de limites. Tout d'abord, le secteur de la pomme est une niche de marché dans l'agro-alimentaire. Il n'est pas évident de généraliser dans d'autres contextes économiques les retombées de cette recherche, notamment dans des filières agricoles comportant de nombreux intermédiaires comme dans l'élevage, ou dans des filières plus concentrées au niveau de l'offre comme dans les céréales.

En conséquence, la manière de répondre au défi de l'agriculture durable peut changer d'un secteur d'activité à l'autre, sans que le modèle de la pomme Ariane ${ }^{\varpi}$ ne serve de référence universelle, d'autant plus que ce modèle n'a pas été actualisé avec des données empiriques récentes. Pour renforcer la validité externe de ce modèle, il conviendrait de s'interroger sur les évolutions dans la filière de la pomme Ariane ${ }^{\varpi}$, pour observer si l'INRA conserve toujours un rôle central d'acteur-réseau et si les différents maillons de la filière parviennent toujours à préserver leur bien commun : la rente collective à partir de l'innovation technologique. 
Lancienneté des données interroge donc sur la pérennité du modèle d'organisation en réseau présenté dans la recherche mais pas sur la contribution exploratoire. A partir de notre recherche, nous sommes convaincus que les grandes questions de civilisation sur le réchauffement climatique, la crise alimentaire, la surpopulation urbaine, la disparition de la biodiversité, trouveront des réponses dans l'innovation technologique issue du rapprochement public-privé, en dehors des cadres traditionnels de l'Etat et du marché.

\section{Bibliographie}

Arrow K. (1970). Social Choice and Individual Values, Second edition, Yale University Press.

Assens C. (2013). Le management des réseaux : tisser du lien social pour le bien être économique, Editions de Boeck.

Association nationale pommes poires (2016) : La pomme en France (http://www.medfel.com/fr/presentation/zones-de-production-exposantes, consulté le 23 juin 2016).

Astley W.G.; Fombrun C.J. (1983). «Collective strategy : social ecology of organizational environments ", Academy of Management Review, Vol. 8, n4, p. 576-587.

Baumol W, Bowen W (1966). New York Performing Arts: The Economic Dilema, MIT Press.

Buchanan J. (1965). «An Economic Theory of Clubs», Economica, Vol. 32, n 125, p. 1-14.

CoAse R. (2005). L'entreprise, le marché et le droit, Editions d'Organisation.

Coléno F.C.; Ould Ahmed S.M. (2009). «Technical and organizational innovation : a collaboration between private and public sector, the case of the Ariane ${ }^{\circledast}$ apple» Proceeding of the 17th congress of the International Farm Management Association, Bloomington (USA), 19-24 July Vol. 2. p. 189-198

DALPÉ, R. (2003). «Interaction between public research organizations and industry in biotechnology», Managerial and Decisions Economics, Vol. 24, n²-3, p. 171-185.

Desquilbet M.; Guyomard H. (2002). «Taxes and subsidies in vertically related markets», American Journal of Agricultural Economics, Vol. 84, nº p. p. 1033-1041.

Garmendia, L., Grillet, E., Lafond, S., 2009, Le partenariat INRA-CEP INNOVATION-NOVADI : Bases contractuelles et stratégies associées, Innovations Agronomiques, 7, p. 179-184.

HannaCHI, M.; COLÉNo F.C. (2015). «Managing the evolution of coopétition dynamics : Alongitudal case study on the French grain merchant industry». Business and Management Research. Vol. 4, n4 4 p. 13-26.

GeIndre, S. (2005). «Le rôle de l'acteur tiers dans la construction d'un réseau stratégique», Revue Française de Gestion, Vol. 31, $\mathrm{n}^{\circ} 154$, p. $75-91$.

HanNaChi M. (2011). «La coopétition au service du bien commun. Les stratégies des entreprises de collecte et de stockage de céréales face aux OGM.» Thèse de doctorat de l'Université Versailles Saint-Quentin-En-Yvelines. 251p.

Hannachi, M.; Coléno, F.C.; Assens, C. (2010). «La collaboration entre concurrents pour gérer le bien commun : le cas des entreprises de collecte et de stockage de céréales d'Alsace», Gérer et Comprendre. Vol. 101, p. 16-25
Hardin G. (1968). «The Tragedy of the Commons», Science, Vol. 162, n³859, p. 1243-1248.

Hatchuel A.; Segrestin B. (2007). «La société contre l'entreprise? vers une norme d'entreprise à progrès collectif», Droit et Société, Vol. 65, p. 27-40.

JICK T.D. (1979). «Mixing qualitative and quantitative methods : triangulation in action", Administrative Science Quarterly, Vol. 24, nº 4 , p. 602-611.

Labatut, J. (2009). "Gérer des biens communs. Processus de conception et régimes de coopération dans la gestion des ressources génétiques animales». Thèse de doctorat de l'Ecole Nationale Supérieure des Mines de Paris. 382. p.

Link A.N.; ReEs, J. (1990). «Firm size, University based research, and the returns to R\&D."Small Business Economics Vol. 2, $\mathrm{n}^{\circ} 1, \mathrm{p} .25-31$.

Loilier T.; Tellier A. (1999). Gestion de l'innovation, Editions Management et Société, Caen.

McDonald B.A.; Linde C. (2002). «Pathogen population genetics, evolutionary potential, and durable resistance». Annual Review of Phytopathology, Vol. 40, p. 349-379.

Musgrave, R. (1959). A theory of public finance, Mc Graw Hill.

Ostrom E. (2010). Gouvernance des biens communs - Pour une nouvelle approche des ressources naturelles, De Boeck Editions.

Ostrom E. (2005). Understanding institutional diversity, Princeton University Press.

Ould Hamed S.M. (2008). «Stratégies collectives pour la gestion de la durabilité des résistances variétales ", Mémoire de Master, ISM- Université de Versailles Saint-Quentin en Yvelines. 99 p. + annexes.

SAко, M. (1996). «Supplier's associations in the japonese automobile industry : collective action for technology diffusion ", Cambridge Journal of Economics, Vol. 20, nº 6, p. 651-671.

Samuelson P. (1954). "The Pure Theory of Public Expenditure», Review of Economics and Statistics, Vol. 36, n 4 , p. 387-389.

Savary, S.; Ficke A.; Aubertot, J.N.; Hollier, C. (2012). «Crop losses due to diseases and their implications for global food production losses and food security». Food Security Vol. 4, nº, p. 519-537.

Smith, A. (1776). Recherches sur la nature et les causes de la richesse des nations, Glasgow University Press.

Tinbergen, J. (1952). Techniques modernes de la politique économique, Dunod.

Torre A. (2002). «Les AOC sont-elles des clubs? Réflexions sur les conditions de l'action collective localisée, entre coopération et règles formelles ", Revue d'Economie industrielle, $\mathrm{n}^{\circ} 100$, pp. 39-62.

United Nations Organization (1992). Conference on Environment \& Development, Rio de Janerio, Brazil, 3 to 14 June.

YIN R. (1989). Case study research: Design and methods (Rev. ed.). Newbury Park, CA : Sage Publishing. 166p.

Zhu, Y.Y.; Chen, H.R.; Fan, J.H.; Wang, Y.Y.; LI, Y.; Chen, J.B.; FAN, J.X.; YANG, S.S.; Hu, L.P.; Leung, H.; Mew, T.W.; Teng, P.S.; WAnG, Z.H.; MundT, C.C. (2000.) «Genetic diversity and disease control in rice." Nature Vol. 406, ${ }^{\circ} 6788$, p. $718-722$. 